\title{
Rheumatoid Arthritis Management for General Internists
}

\author{
Mitchell Uh MD FRCPC, David Collins MD FRCPC ABIM
}

\begin{abstract}
About the Authors
Mitchell Uh is a rheumatology fellow at the University of British Columbia (UBC), in Vancouver, British Columbia. David Collins is a clinical assistant professor at UBC and works at Vancouver General Hospital. Correspondence may be directed to davidcollins911@hotmail.com.
\end{abstract}

$\mathrm{R}_{\mathrm{h}}^{\mathrm{s}}$ heumatoid arthritis (RA) is a chronic systemic inflammatory disorder that causes progressive joint destruction, deformity, and significant disability. In addition to arthritis, RA is associated with numerous extra-articular features with attendant morbidity and mortality. These include but are not limited to ocular inflammation, interstitial lung disease, pleuropericardial disease, vasculitis, accelerated atherosclerosis, and increased risk of lymphoma. The prevalence of RA in the Canadian population is approximately $1 \%{ }^{1}$

All patients with RA should have a multidisciplinary treatment approach, referral to a specialist, and early therapy with disease-modifying anti-rheumatic drugs (DMARDs). Effective treatment with DMARDs slows the progression of joint destruction, reduces extra-articular manifestations, and reduces mortality. ${ }^{2-4}$ Wait-lists to see a rheumatologist are often in excess of 6 months, despite efforts to prioritize patients with suspected RA by triaging. In larger centres, early inflammatory arthritis clinics have actually been developed to triage these patients more quickly, with the intent of starting DMARDs sooner. Despite these efforts, the median time from symptom onset to appropriate treatment with DMARDs is over 8 months, with the majority of the delay due to events prior to rheumatology referral. $^{5}$

It is therefore paramount that earlier identification and appropriate therapy of RA be undertaken by primary care providers and general internists, as they may be able to see such patients sooner or may be performing the bulk of rheumatology care in underserviced areas. This is particularly likely to happen in mid-sized communities where the general internist functions as the local specialist. Historically, there have been numerous DMARD choices available. These options, combined with the proliferation of biologic molecules over the past decade, have made for sometimes confusing choices when it comes to therapeutic decision making. Recently, the Canadian
Rheumatology Association (CRA) published evidence-based guidelines for the evaluation, monitoring, and treatment of adult RA. ${ }^{6,7}$ The following recommendations, unless otherwise noted, have been adapted from the recent CRA guidelines.

\section{Initial DMARD Treatment}

Patients with persistent synovitis should have DMARD therapy initiated as soon as possible. Joint damage begins early on, and disease duration is inversely correlated with response to therapy. ${ }^{8}$ Generally, methotrexate should form the backbone of initial therapy, unless there are contraindications. Prior to treatment, patients should undergo a complete blood count, liver and kidney function tests, chest radiography, and be assessed for chronic hepatitis B and C and human immunodeficiency virus where indicated. The dose of methotrexate should be maximized, up to $25 \mathrm{mg}$ weekly orally or subcutaneously. Those with gastrointestinal intolerance to the oral formulation may benefit from the parenteral form. In fact, many rheumatologists are promoting this preferentially to their patients as the subcutaneous injection can be self-administered. It is generally considered superior to the oral route. There has been a great variation in how to start and titrate up methotrexate, but with proper monitoring we are becoming more aggressive, especially in severe disease. Depending on comfort levels, rheumatologists might even start a patient right at $25 \mathrm{mg} / \mathrm{wk}$, a dosage that was previously unheard of. It should be noted that methotrexate is a useful treatment in other forms of inflammatory arthritis, such as in psoriatic arthritis or arthritis associated with connective tissue disease, so it remains a good choice in patients in whom the diagnosis may be initially unclear. ${ }^{910}$

Patients with RA should be assessed for poor prognostic factors and, if they exist, be considered for initial combination DMARD therapy. Poor prognostic factors include high swollen and/or tender joint counts, extra-articular features, poor 
functional status, early erosive disease, high inflammatory markers, and rheumatoid factor or anti-cyclic citrullinated peptide (anti-CCP) antibody positivity. Smokers should be counselled on smoking cessation as it is associated with higher disease activity and more difficult-to-treat disease. ${ }^{11,12}$

Combinations with proven efficacy greater than methotrexate alone include methotrexate, sulfasalazine, and hydroxychloroquine; methotrexate and cyclosporine; methotrexate and gold; and methotrexate and leflunomide. ${ }^{13-16}$ Particular care should be taken with the last combination as the incidences of gastrointestinal intolerance, transaminitis, and leukopenia increase with its use. Dosage reductions may be necessary to minimize side effects. Combinations not including methotrexate are sometimes necessary and can be considered on an individual basis.

DMARDs have a slow onset and generally do not show their maximum benefit until 8-12 weeks of use. Corticosteroids are acceptable in the short term in combination with DMARDs for bridging therapy, to control symptoms more rapidly, or while awaiting specialist assessment. They should be reserved for people with high disease activity, used at the lowest dosage possible, and tapered as quickly as safely possible. Generally, steroids are reserved as a "quick fix" for a patient whose function is really suffering. One concern that the general internist might have when debating whether to start steroids or not is, "Will I mask the symptoms and therefore the diagnosis for when the patient is assessed by a rheumatologist?" But with wait-lists and access as difficult as they are, most rheumatologists understand the decision to prescribe steroids, and they take the history and response to steroids into account in their assessment. It helps to document the existence of tender or swollen joints in the referral letter.

Another valid concern is, "Will I end up with the patient 'trapped' on steroids?" The long-term side effects of steroids are numerous, and we all try to minimize the dosage by using DMARDs. For this reason, one should generally reserve steroids for the patient whose physical function is truly suffering.

The CRA does not make specific recommendations on the use of nonsteroidal anti-inflammatory drugs (NSAIDs). While they sometimes provide modest pain relief, they have not been shown to slow disease progression or prevent joint damage in RA. They should be used cautiously, weighing their benefit against the increased renal, gastrointestinal, and cardiovascular toxicity, especially in the long term, given the already increased cardiovascular risk in patients with RA. NSAIDs should not be considered a substitute for DMARDs.

\section{Monitoring}

Clinical assessment of RA should be performed every 1-3 months, with longer intervals for those with stable low disease activity or in remission. In practice, patients on methotrexate or leflunomide should have complete blood counts and renal and liver function tests assessed monthly to monitor for adverse effects. Changes in therapy should be considered for those experiencing adverse effects, or with ongoing disease activity despite an adequate trial of DMARD therapy. It may be here that a general internist can work with a consulting rheumatologist to follow up with the patient. If a laboratory abnormality does occur during monitoring, and the general internist is uncertain what actions to take, then this is where most rheumatologists do not mind fielding a phone call to assist with a patient known to them, or granting a follow-up sooner than had been scheduled.

\section{Changes in Treatment}

The goal of RA treatment is disease remission or low disease activity if remission is not possible. Therapy should be adjusted every 3-6 months to attain this goal. If on monotherapy, another DMARD or combination therapy should be considered. The options are as above, with the choice depending on the individual patient's status and preference. Patients with persistently moderate or high disease activity despite treatment with two or more DMARDs (including methotrexate) alone or in combination for at least 3 months should be considered for biologic therapy. Each province or territory has different requirements for biologic therapy funding, and these should be referred to when applying for coverage on behalf of patients. This is really the "art" of rheumatology. With core internal medicine training, it might be difficult to gain adequate confidence in monitoring or changing DMARDs. Some internists may do extra training or take an interest in this area and become comfortable with certain medications; but if in doubt, they should defer to the consulting rheumatologist for changes.

\section{Biologics}

All patients being considered for biologic therapy should be referred to an experienced specialist. For most, this will be a rheumatologist, though some general internists also have experience with biologic medications. The choice and administration of specific therapy is beyond the scope of this article, but certain considerations should be made by all physicians involved in RA care prior to initiating and during biologic therapy in patients.

In the absence of contraindications, patients should remain on methotrexate concurrently with a biologic. In general, methotrexate increases the efficacy, promotes longevity, and 
reduces the adverse reactions of biologics compared with monotherapy. ${ }^{17}$

The risk of reactivating latent tuberculosis infection (LTBI) is increased with most biologics. All patients should be screened for the risk of LTBI by history, physical examination, chest radiography, and a tuberculin skin test. Those with a positive skin test should have active TB ruled out. Interferon- $\gamma$ release assay may be indicated in patients with prior bacille CalmetteGuérin with low clinical suspicion of LTBI who have a positive skin test. All patients considered to have LTBI should be treated appropriately. Biologic therapy can be started 1 month following the initiation of LTBI therapy in patients adherent to therapy.

RA patients should be screened for chronic hepatitis B and $\mathrm{C}$ prior to biologic therapy. There is a paucity of evidence in the area, but there is suggestion that the risk of hepatitis $B$ reactivation, especially, is increased with the use of tumour necrosis factor $\alpha$ (TNF- $\alpha$ ) inhibitors. ${ }^{18,19}$ Treatment of such patients is still possible in some situations, usually with cotreatment of hepatitis $\mathrm{B}$, and in close consultation with a hepatologist. Treatment strategies need to be formulated carefully on a case-by-case basis.

If possible, vaccinations should be administered prior to the initiation of methotrexate or biologic therapy. The immune response to inactivated vaccinations may be attenuated while on these therapies, reducing their effectiveness. The CRA recommends that patients receive scheduled influenza and pneumococcal vaccinations during biologic therapy, as respiratory infections are some of the most common experienced by patients with RA. ${ }^{20}$ Hepatitis $B$ vaccination can be given to high-risk individuals. Here, a thorough internist might actually be able to complement a patient's care if the rheumatologist and general practitioner have overlooked the vaccinations.

Live vaccinations carry the theoretical risk of causing disease in patients on biologics. The CRA recommends that patients receive live vaccines $2-4$ weeks prior to initiating therapy with biologics, or interrupt treatment for 5 half-lives if it is necessary to administer a live vaccine. The length of biologic interruption varies with the half-life of the medication (ranging from 21.5 days for etanercept to 105 days for rituximab). In situations where this is not feasible, the risk of developing disease by not vaccinating needs to be weighed against the risk of causing disease by vaccinating. The herpes zoster vaccine should be considered in patients with RA who are over age 60 years and other high-risk groups, following the above recommendations for live vaccines.

The effects of biologics in malignancy remain controversial. Clinical trials with biologics (with the exception of rituximab) have excluded cancer patients; however, long-term registry data seem to indicate that TNF- $\alpha$ inhibitors do not appear to increase the risk of cancer, with the exception of nonmelanoma skin cancers. ${ }^{21,22}$ Treatment with all DMARDs and biologics should be held in the setting of active malignancy and during therapy for malignancy. The chemotherapeutic agents used often obviate the need for specific DMARDs. In patients with a history of lymphoma, hydroxychloroquine, sulfasalazine, and rituximab may be used; TNF- $\alpha$ inhibitors should not be used; and other DMARDs and biologics should be used with caution. In patients with a history of solid malignancy or nonmelanoma skin cancer, traditional DMARDs may be used and biologics should be used with caution. All decisions regarding therapy should be made in close consultation with the patient and the oncologist.

Patients with RA often undergo surgical procedures for joint replacements. Ideally, patients should have well-controlled disease prior to undergoing any procedure, as uncontrolled disease is associated with poor perioperative outcomes. ${ }^{23}$ This should be balanced against the possible increased risk of perioperative infection in immunosuppressed patients. Methotrexate can safely be continued without interruption perioperatively. For the biologics, it is recommended that they be held for $2-5$ half-lives prior to surgery, depending on the type of surgery and the status of the patient. Biologics can be resumed postoperatively if there is no evidence of infection and wound healing is adequate.

The advent of RA therapy over the past decade has revolutionized the treatment goals and expectations in this chronic, progressive, and potentially disabling disease. Outcomes have included reduced morbidity and mortality, and increased function. With the newer therapies have come new challenges and questions, many of which remain unanswered. The CRA guidelines presented here have consolidated most of the quality evidence to provide a road map in the increasingly complex world of modern RA therapy. Many of the issues addressed are commonly encountered in general medicine, and these guidelines should help to expedite and standardize RA management across the country.

\section{References}

1. The Arthritis Society. Rheumatoid arthritis. Toronto (ON): The Society, 2011; http://www.arthritis.ca/document.doc?id=336. Accessed November $2,2012$.

2. Choi HK, Hernan MA, Seeger JD, et al. Methotrexate and mortality in patients with rheumatoid arthritis: a prospective study. Lancet 2002;359:1173-7.

3. Weinblatt ME, Kaplan H, Germain BF, et al. Methotrexate in rheumatoid arthritis. A five-year prospective multicenter study. Arthritis Rheum 1994;37:1492-8. 
4. Westlake SL, Colebatch AN, Baird J, et al. The effect of methotrexate on cardiovascular disease in patients with rheumatoid arthritis: a systematic literature review. Rheumatology (Oxford) 2010;49:295-307.

5. Tavares R, Pope JE, Tremblay JL, et al. Time to disease-modifying antirheumatic drug treatment in rheumatoid arthritis and its predictors: a national, multicenter, retrospective cohort. J Rheumatol 2012;39:2088-97.

6. Bombardier C, Hazlewood GS, Akhavan P, et al. Canadian Rheumatology Association recommendations for the pharmacological management of rheumatoid arthritis with traditional and biologic disease-modifying antirheumatic drugs: part II safety. J Rheumatol 2012;39:1583-602.

7. Bykerk VP, Akhavan P, Hazlewood GS, et al. Canadian Rheumatology Association recommendations for pharmacological management of rheumatoid arthritis with traditional and biologic disease-modifying antirheumatic drugs. J Rheumatol 2012;39:1559-82.

8. Anderson JJ, Wells G, Verhoeven AC, Felson DT. Factors predicting response to treatment in rheumatoid arthritis: the importance of disease duration. Arthritis Rheum 2000;43:22-9.

9. Lie E, van der Heijde D, Uhlig T, et al. Effectiveness and retention rates of methotrexate in psoriatic arthritis in comparison with methotrexatetreated patients with rheumatoid arthritis. Ann Rheum Dis 2010;69:671-6.

10. Fortin PR, Abrahamowicz M, Ferland D, et al. Steroid-sparing effects of methotrexate in systemic lupus erythematosus: a double-blind, randomized, placebo-controlled trial. Arthritis Rheum 2008;59:1796-804.

11. Canhao H, Rodrigues AM, Mourao AF, et al. Comparative effectiveness and predictors of response to tumour necrosis factor inhibitor therapies in rheumatoid arthritis. Rheumatology (Oxford) 2012;51:2020-6.

12. Fisher MC, Hochberg MC, El-Taha M, et al. Smoking, smoking cessation, and disease activity in a large cohort of patients with rheumatoid arthritis. J Rheumatol 2012;39:904-9.

13. O'Dell JR, Leff R, Paulsen G, et al. Treatment of rheumatoid arthritis with methotrexate and hydroxychloroquine, methotrexate and sulfasalazine, or a combination of the three medications: results of a two-year, randomized, double-blind, placebo-controlled trial. Arthritis Rheum 2002;46:1164-70.

14. Stein CM, Pincus T, Yocum D, et al. Combination treatment of severe rheumatoid arthritis with cyclosporine and methotrexate for forty-eight weeks: an open-label extension study. The Methotrexate-Cyclosporine Combination Study Group. Arthritis Rheum 1997;40:1843-51.
15. Lehman AJ, Esdaile JM, Klinkhoff AV, et al. A 48-week, randomized, double-blind, double-observer, placebo-controlled multicenter trial of combination methotrexate and intramuscular gold therapy in rheumatoid arthritis: results of the METGO study. Arthritis Rheum 2005;52:1360-70.

16. Kremer JM, Genovese MC, Cannon GW, et al. Concomitant leflunomide therapy in patients with active rheumatoid arthritis despite stable doses of methotrexate. A randomized, double-blind, placebo-controlled trial. Ann Intern Med 2002;137:726-33.

17. Nam JL, Winthrop KL, van Vollenhoven RF, et al. Current evidence for the management of rheumatoid arthritis with biological disease-modifying antirheumatic drugs: a systematic literature review informing the EULAR recommendations for the management of RA. Ann Rheum Dis 2010;69:976-86.

18. Wendling D, Auge B, Bettinger D, et al. Reactivation of a latent precore mutant hepatitis B virus related chronic hepatitis during infliximab treatment for severe spondyloarthropathy. Ann Rheum Dis 2005;64:788-9.

19. Ostuni P, Botsios C, Punzi L, et al. Hepatitis B reactivation in a chronic hepatitis B surface antigen carrier with rheumatoid arthritis treated with infliximab and low dose methotrexate. Ann Rheum Dis 2003;62:686-7.

20. van Assen S, Elkayam O, Agmon-Levin N, et al. Vaccination in adult patients with auto-immune inflammatory rheumatic diseases: a systematic literature review for the European League Against Rheumatism evidencebased recommendations for vaccination in adult patients with autoimmune inflammatory rheumatic diseases. Autoimmun Rev 2011;10:34152.

21. Abasolo L, Judez E, Descalzo MA, et al. Cancer in rheumatoid arthritis: occurrence, mortality, and associated factors in a South European population. Semin Arthritis Rheum 2008;37:388-97.

22. Raaschou P, Simard JF, Neovius M, Askling J. Does cancer that occurs during or after anti-tumor necrosis factor therapy have a worse prognosis? A national assessment of overall and site-specific cancer survival in rheumatoid arthritis patients treated with biologic agents. Arthritis Rheum 2011;63:1812-22.

23. Pieringer H, Stuby U, Biesenbach G. Patients with rheumatoid arthritis undergoing surgery: how should we deal with antirheumatic treatment? Semin Arthritis Rheum 2007;36:278-86. 